\title{
Ward round accreditation: an innovative quality improvement project to develop and improve the existing medical ward round at Warrington Hospital
}

\author{
Authors: Lisa Waters, ${ }^{A^{*}}$ Alex Crowe, ${ }^{B}$ Katie Ethell ${ }^{C}$ and Ammar Ahmed ${ }^{C}$
}

\begin{abstract}
Introduction
Medical ward rounds have long been considered the centrepiece of inpatient care. They provide an opportunity for high quality, safe, multidisciplinary patient review while facilitating shared decision making, patient flow and important educational input. Variation in ward round structure is inevitable and there is always a fine line between balancing service needs and providing education. In 2012 the Royal College of Physicians (RCP) and Royal College of Nursing (RCN) produced Ward rounds in medicine: principles for best practice, providing core recommendations and best practice for conducting ward rounds. ${ }^{1}$ Accreditation of clinical departments and processes is increasingly being utilised in standardising best practice in clinical care. Here, for the first time, we outline how we are developing an accreditation process at Warrington Hospital with the aim of providing a framework for a consistent yet flexible, efficient and effective multidisciplinary, patient and educationfocused medical ward round.
\end{abstract}

\section{Materials and methods}

In this quality improvement project undertaken at a district general hospital we employed both qualitative and quantitative data collection methods to establish current ward round practice. Junior doctors collected real time ward round data including compliance with recognised metrics such as venous thromboembolism (VTE) prophylaxis assessment, ceiling of care discussion and expected date of discharge (EDD). Educational value of ward rounds was collated via a questionnaire with opportunity to record any assessments undertaken and provide suggestions for improving the experience. A ward round Standard Operating Procedure was drawn up.

Exploring stakeholders' perspectives, we surveyed inpatients. Prospectively, using the data collected we aim to implement metrics and an accreditation award system for medical ward rounds devised from our data collection, RCP/RCN guidance and best practice. Once standards are established, we will implement an accreditation process for each metric, generating ward round accreditation for the organisation.

\section{Results and discussion}

Nineteen patients were surveyed with a median age of 48 across a range of socioeconomic groups. $90 \%$ understood the term ward round. There were mixed responses to who should lead a ward round ( $22 \%$ said nurses, $63 \%$ said doctors and $15 \%$ did not mind). $89 \%$ of patients were aware of their diagnosis. $42 \%$ were aware of their EDD. $100 \%$ were agreeable to teaching taking place on a ward round. $37 \%$ felt the round required improved communication.

Seven individual consultant-led rounds and 37 patient interactions were observed. $46 \%$ of VTE assessments were completed. $100 \%$ of observation charts were reviewed. $95 \%$ of prescriptions were reviewed. EDD was documented in $51 \%$ of patients. $11 \%$ had a ceiling of care documented. Teaching occurred on $57 \%$ of rounds. One round provided opportunity for web-based placed assessments.

\section{Conclusion}

This project has engaged multiple stakeholders to demonstrate that there is currently considerable variation in medical ward rounds undertaken across the trust and that standardisation with the implementation of set metrics provide improved patient care, flow and enhanced junior doctor educational experience. We aim to roll out implementation of an accreditation system to allow us to demonstrate improvements in compliance with RCP/RCN guidance and thus provide a systematic, consistent, multidisciplinary, education-filled medical ward round for all.

\section{Reference}

1 Royal College of Physicians, Royal College of Nursing. Ward rounds in medicine: principles for best practice. London: RCP, 2012. 